\title{
Preffi 2.0: un outil néerlandais pour analyser l'efficacité des interventions en promotion de la santé
}

Quels sont les éléments qui rendent les programmes de promotion de la santé efficaces? Comment peut-on concevoir et mettre en œuvre des projets de façon à créer les conditions optimales à leur efficacité? Au cours des 20 dernières années, de nombreux chercheurs ont essayé de répondre à ces questions en effectuant des études centrées sur la méta-analyse de l'efficacité des programmes et en recourant de préférence à des essais contrôlés randomisés (ECR) pour en évaluer l'efficacité. Ils ont découvert que les programmes de promotion de la santé ne sont pas tous fondés sur des preuves d'efficacité, loin s'en faut. Les effets significatifs de nombreux programmes ont certes été démontrés dans plusieurs pays, mais en moyenne l'ampleur de ces effets a jusqu'à présent été modérée (Kok

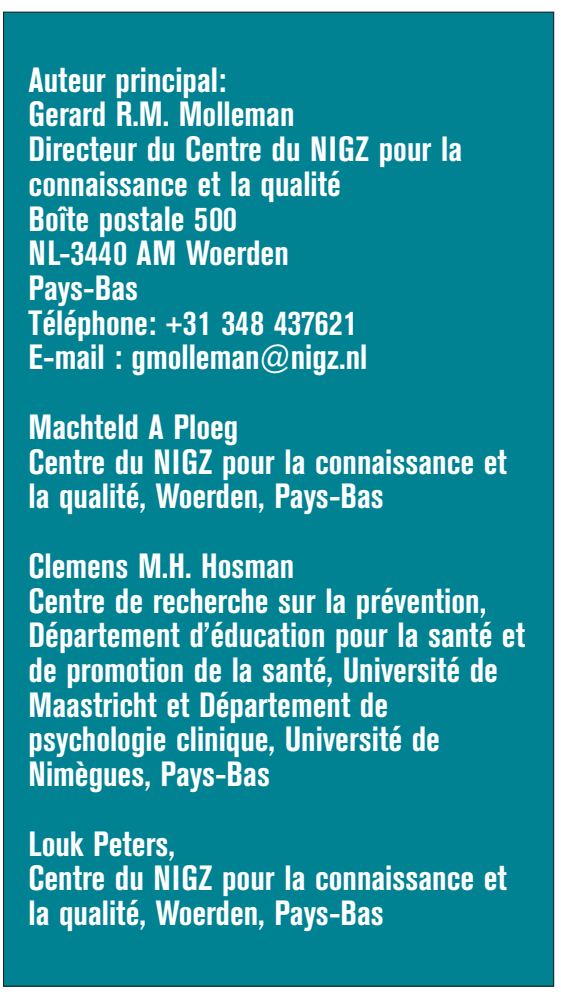

et al., 1997, Durlak et Welsh, 1997, Boddy, 1999). Par ailleurs, alors qu'il s'est avéré que de nombreux programmes sont efficaces ou moyennement efficaces, l'efficacité de nombreux autres laissait à désirer, voire était absente.

Il a également été établi que bon nombre des programmes estimés efficaces ne l'étaient que pour une partie du groupe cible, uniquement pour un nombre limité d'objectifs ou seulement à court ou à moyen terme, ou alors qu'ils perdaient une partie ou la totalité de leur efficacité dans un contexte différent. Cela signifie qu'il reste de la place pour l'amélioration de la qualité et de l'efficacité des programmes. Ces diverses études sur les effets des programmes de promotion de la santé ont sans nul doute produit quantité d'informations, mais leurs résultats sont rarement appliqués dans la pratique de la promotion de la santé. Les praticiens trouvent qu'il est difficile d'évaluer la valeur des résultats de la recherche qui parfois sont même contradictoires. Par ailleurs, les chercheurs négligent souvent de donner des détails sur les conditions contextuelles (en terme de temps, de financement et de soutien disponible) et les circonstances (contexte local, influence sociale, culturelle et économique, moment) dans lesquelles les effets signalés ont été obtenus, alors que ces détails sont nécessaires pour décider s'il faut adapter l'intervention pour qu'elle puisse être répliquée ou mise en œuvre avec succès dans une situation différente, et la façon de procéder.

Chercheurs et praticiens travaillent souvent selon des échéanciers différents. Les tentatives d'amélioration de l'efficacité au moyen d'ECR exigent des investissements à long terme et comportent de longs délais entre l'élaboration d'un programme et la production d'information sur ses effets. Ce processus est important mais extrêmement lent. Les décideurs et praticiens en revanche ont souvent besoin de recevoir l'information plus rapidement, puisqu'on leur demande de réagir à des problèmes précis à brève échéance, sous forme de programmes et d'interventions qui produisent un maximum d'effet. En bref, ce dont ils ont besoin, c'est que les informations obtenues grâce à la recherche soient traduites en recommandations pratiques qui correspondent au contexte dans lequel ils doivent travailler.

Ceci signifie que pour stimuler une promotion de la santé efficace, il faut à la fois :

1. élaborer et diffuser des programmes dont l'efficacité a été démontrée; et 2. informer sur les principes d'efficacité qui influencent l'efficacité de la promotion de la santé dans la pratique, et la façon dont les professionnels utilisent ces informations.

C'est sur cette toile de fond que l'Institut néerlandais de promotion de la santé et de prévention des maladies (NIGZ) a lancé le projet Preffi au milieu des années 90. Le projet est un processus à long terme par lequel on tente d'améliorer l'efficacité de la promotion de la santé en stimulant une réflexion systématique et critique sur les programmes et les projets.

Mots clés
- efficacité
- Pays Bas
- outils d'évaluation
- programmes de promotion
de la santé


Le pivot de ce projet est le Preffi, un outil consistant en un ensemble de recommandations sur les éléments qui contribuent à déterminer l'efficacité de projets de prévention et de promotion de la santé et qui prennent en compte la connaissance scientifique disponible sur les effets escomptés ainsi que des informations émanant d'échanges avec les praticiens au sujet de ces indicateurs.

Dans cet article, les auteurs décrivent l'élaboration et le contenu de la seconde version de cet outil, le Preffi 2 . ; Ils se basent sur des données qui ont été réunies systématiquement auprès des utilisateurs de l'outil à diverses étapes de son élaboration et de son utilisation (Molleman et al., 2004).

\section{Élaboration de Preffi}

L'élaboration systématique des recommandations du Preffi a commencé en 1994 ; elle s'est fondée sur les résultats de recherche alors disponibles, sur un examen des méthodes

d'élaboration de recommandations et sur de nombreuses entrevues avec des praticiens. Ce processus a abouti en 1995 au lancement de la première version de Preffi, Preffi 1.0, alors baptisé " outil de stimulation de l'efficacité de la promotion de la santé " (Molleman, 1999).

En 2000, un groupe de travail mixte composé de membres du NIGZ et du Centre de recherche sur la prévention de l'Université de Nimègues a commencé à élaborer une deuxième version de Preffi en s'appuyant sur l'expérience acquise grâce à Preffi 1.0 depuis son lancement. L'équipe a essayé d'améliorer quatre aspects : le contenu, les normes, le format et le positionnement. Pour ce qui est du contenu, il s'agissait d'intégrer dans la deuxième version les nouvelles informations fournies par la recherche et la pratique en promotion de la santé. Il fallait en outre opérationnaliser les critères de Preffi de façon à ce que les utilisateurs puissent comparer leurs propres projets par rapport à des normes établies et que des tiers puissent évaluer un projet. Le format élaboré pour la nouvelle version de Preffi voulait aussi rendre justice à la nature cyclique et itérative de nombreux projets de promotion de la santé. Enfin, on a jugé nécessaire de préciser le positionnement de Preffi en tant qu'outil de contrôle de qualité destiné à déterminer et à améliorer les conditions propices à l'efficacité d'un projet. Par conséquent, il fut décidé de modifier son nom et de l'appeler "Outil de gestion des effets de la promotion de la santé ".

Pour élaborer Preffi 2.0, de nombreux efforts ont été consentis pour développer une base scientifique solide et rendre les critères de Preffi opérationnels. Pour cela, nous avons travaillé en étroite collaboration avec un comité scientifique représentant les divers organismes impliqués dans la recherche et la promotion de la santé aux Pays-Bas. Par ailleurs, un comité de praticiens, constitué de 53 professionnels néerlandais de la promotion de la santé, garantissait que la perspective des utilisateurs n'était pas négligée.

Le processus d'élaboration de Preffi 2.0 s'est basé sur une approche consistant en un certain nombre d'étapes systématiques et a utilisé diverses méthodes d'évaluation formatives axées sur les produits, les experts ou le groupe cible (Jong et Schellens, 2000). Dans la méthode axée sur les produits, ce sont les concepteurs eux-mêmes qui déterminent les aspects sur lesquels ils fondent leur évaluation, tandis que dans le cadre des deux autres méthodes, le concept est soumis à des experts externes et à des membres du groupe cible respectivement. Le processus de conception de Preffi 2.0 a commencé par la méthode axée sur les produits (étapes 1 à 5), suivie de celle axée sur les experts (étapes 4 à 6) et enfin de celle sur le groupe cible (étapes 6 à 7).

Étape 1. Le groupe de travail Preffi a tout d'abord essayé de déterminer comment l'outil allait se positionner dans le contexte général de la gestion de qualité. Il a décidé que, pour ce qui est des extrants du moins à cette étape de son élaboration, Preffi devait se concentrer sur l'efficacité, la pertinence et la couverture des programmes plutôt que sur d'autres caractéristiques telles que le rapport coût-efficacité ou la satisfaction des clients. Sur le plan des intrants, Preffi devait être axé sur les processus opérationnels réels intervenant dans la conception et la mise en œuvre des programmes. Des variables importantes du contexte, notamment les aspects structurels des organisations (infrastructure, politiques institutionnelles, dotation en personnel, relations de collaboration, etc.) devaient être aussi incluses dans Preffi comme conditions contextuelles importantes.

Étape 2. Il fut décidé que le principe de structuration générale pour les processus opérationnels serait celui d'une approche systématique, puisque tout le monde s'accorde pour reconnaître qu'une approche systématique améliore l'efficacité des programmes de prévention

(Bartholomew et al., 2001, Glanz et al., 2002). Tout comme la nécessité de considérer les conditions contextuelles, les quatre étapes d'une approche systématique (analyse, élaboration, mise en œuvre et évaluation) ont été identifiées comme des dimensions distinctes, et des indicateurs des effets prévus de chacune de ces cinq dimensions ont alors été définis et développés.

Étape 3. Au départ, cinq critères ont été établis pour le choix des indicateurs des effets prévus à savoir la pertinence, l'existence de données scientifiques probantes, la " généralisabilité ", la "modificabilité " et la "mesurabilité ". Lors de l'application de ces critères, nous avons trouvé que pour ainsi dire tous les indicateurs de Preffi 1.0 satisfaisaient aux critères de " généralisabilité " et de "modificabilité ", ce qui signifiait que ces critères n'avaient quasiment aucune valeur discriminatoire, pas plus que la "mesurabilité ", puisque tout indicateur des effets prévus peut, en principe, être rendu clair et mesurable pour le groupe cible des praticiens.

Ceci laissait les critères " pertinence " et " preuves ". Le groupe de travail a décidé que la pertinence était le critère le plus important. On entend par "pertinence " l'effet démontré ou hypothétique d'une caractéristique ou d'une condition particulière d'un projet sur son efficacité.

On a finalement attribué à chaque indicateur d'effet prévu une note en regard de la disponibilité de données scientifiques le concernant afin d'évaluer la nature de Preffi comme outil fondé sur des données probantes (Peters et al., 2003). Une définition large du concept de " preuve " a été utilisée, notamment parce que la valeur de certains indicateurs qui sont réputés être importants est difficile à démontrer dans 
le cadre d'un ECR qui est habituellement considéré comme la forme de données probantes la plus valable. Les principes d'une approche systématique, par exemple, sont habituellement fondés sur une argumentation logique, le consensus et les conclusions de multiples études de cas. Il n'existe pas encore de classement particulier internationalement accepté des types de données probantes, et l'avis selon lequel des méthodes de vérification autres que l'ECR sont également valables recueille de plus en plus de suffrages (McQueen and Anderson, 2002, Koelen et al., 2001).
Étape 4. Les résultats de l'application de ces critères de sélection à Preffi 1.0 ont dans un premier temps été examinés par le groupe de travail, puis individuellement par les membres du Comité scientifique, et finalement tous ensemble. Le résultat de ces consultations a servi à préciser un certain nombre de critères de Preffi 1.0, mais aucun n'a été supprimé. L'idée de l'ajout de nouveaux indicateurs d'effets prévus fondés sur la recherche récente en promotion de la santé, l'analyse des pratiques, ainsi que sur l'expérience acquise avec Preffi 1.0 a plutôt été retenue.

\section{Figure 1}

\section{Modèle de Preffi 2.0}

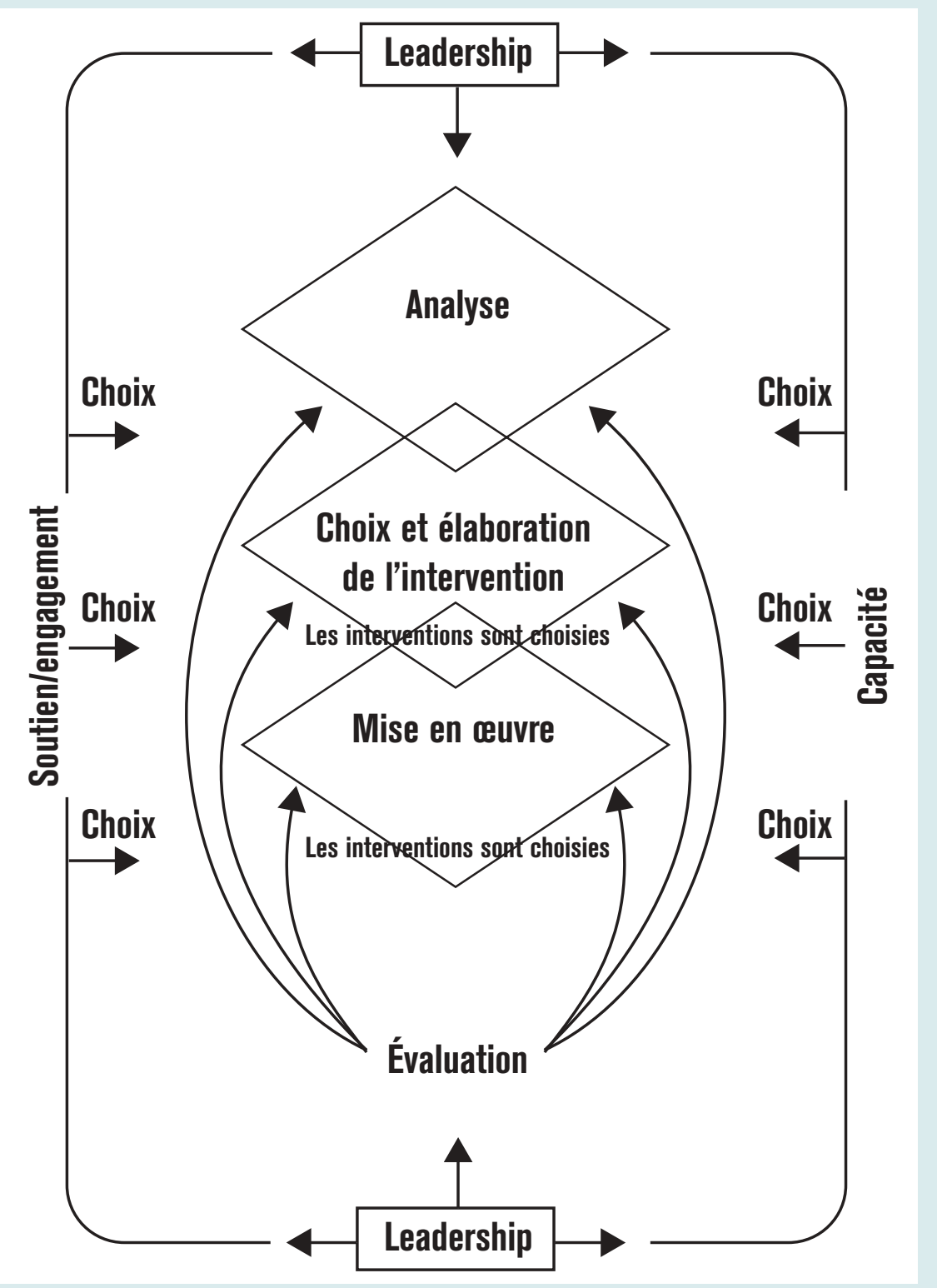

Étape 5. Le choix des indicateurs ainsi effectué a été consigné en détail dans un document explicatif qui décrivait la nature de chaque indicateur, sa relation avec l'efficacité (pertinence) et les données probantes disponibles pour corroborer cette relation. Un second document présentait une opérationnalisation supplémentaire de chaque indicateur par rapport à des questions particulières, ainsi que les normes correspondantes, fondées sur le consensus auquel étaient arrivés, à la suite de discussions, le groupe de travail et le comité scientifique. Ceci devait aider les praticiens à décider dans quelle mesure leur projet tient suffisamment compte de chaque indicateur, tout en proposant des aspects particuliers qui pourraient être améliorés.

Étape 6. En sus du comité scientifique, le comité de praticiens a également été impliqué dans les diverses consultations et a été invité à donner son avis sur les documents présentant le mode d'emploi et de mise en œuvre de Preffi 2.0. Les commentaires reçus ont servi à introduire divers ajustements, notamment comme sous-diviser les catégories d'indicateurs d'effets prévus, en clarifiant les explications et en ajustant le mode opérationnel et les normes.

Étape 7. Une version provisoire de Preffi 2.0 (consistant en un formulaire de pointage, un document explicatif, un document " mode d'emploi " et un manuel de l'utilisateur) a été mise à l'essai pour en valider l'aspect pratique auprès de 35 praticiens aguerris appartenant à un éventail d'institutions. On leur a demandé d'utiliser la version provisoire de Preffi 2.0 pour évaluer deux descriptions de projets, puis de remplir un questionnaire (comportant à la fois des questions ouvertes et fermées) sur leur expérience d'application de l'outil et sur leur avis au sujet de divers éléments de la version provisoire. Des interviews supplémentaires ont été réalisés auprès de 10 des praticiens. Les résultats de ce pré-test ont ensuite été utilisés pour ajuster certains aspects du contenu et de la présentation dans la version finale de Preffi 2.0. de même que préciser son utilisation.

\section{Contenu de Preffi 2.0}

Preffi 2.0 consiste en un ensemble d'outils d'évaluation qui comprend (Molleman et al., 2003) : 
- un formulaire de pointage qui permet aux utilisateurs d'attribuer des notes à une liste de critères de qualité (les fameux indicateurs décrits préalablement), et qui comporte un espace pour décrire les éléments qui doivent être améliorés ; une représentation visuelle du modèle Preffi 2.0 décrit à la section suivante de cet article est également incluse sur ce formulaire ;

- un document intitulé Opérationnalisation et normes qui permet de documenter chaque critère au moyen d'une ou plusieurs questions auxquelles il faut répondre par oui ou par non ; des normes (catégories de notes) pour interpréter les réponses aux questions d'opérationnalisation sont également fournies ;

- un Manuel de l'utilisateur qui explique Preffi 2.0 et donne des instructions pour l'utilisation de l'outil et chacun de ses éléments.

Ceci est complété par un document exhaustif intitulé Guide explicatif qui donne des détails supplémentaires sur les critères de qualité (indicateurs prévisionnels des effets) et examine leur importance pour l'évaluation de l'efficacité ainsi que l'information sur les données probantes disponibles (y compris des références documentaires) (Peters et al., 2003).

\section{Le modèle Preffi 2.0}

Les principaux éléments conceptuels de l'outil sont représentés dans le modèle Preffi 2.0 (voir la figure 1) qui insiste sur la nature dynamique des projets de promotion de la santé, l'interaction constante entre le contenu et les conditions contextuelles et la nature cyclique du processus de promotion de la santé.

L'élément central du modèle montre les étapes qu'il faut suivre dans la conception et la mise en œuvre systématique d'un projet, à savoir: analyse du problème, choix et définition de la bonne intervention, mise en œuvre et évaluation de celle-ci. Les étapes du processus sont représentées par des losanges, parce que chaque étape consiste, en un premier temps, à examiner un vaste ensemble d'options (divergence), puis de choisir parmi ces options en se fondant sur les aspects du contenu et les conditions contextuelles (convergence). Pour donner un exemple, l'analyse du problème consiste, dans le meilleur cas de figure, à déceler toutes les causes/déterminants possibles, après quoi on choisit les déterminants devant être abordés dans le cadre de l'intervention, en se fondant sur des arguments de contenu, de pertinence, de " modificabilité " et de faisabilité pratique. Les losanges se chevauchent parce que le processus de sélection doit toujours prendre en ligne de compte les conséquences et les options de l'étape suivante. Ainsi, lorsqu'ils choisissent une intervention particulière, les concepteurs devraient déjà prendre en considération les possibilités de mise en œuvre. L'évaluation porte sur tous les moments du processus auxquels des choix doivent être faits, et c'est pour cette raison que des flèches pointent vers tous ces moments.

L'efficacité des interventions et les choix qui peuvent être faits dans le cadre de ces interventions sont déterminés en partie par des conditions contextuelles telles que le soutien au projet, les moyens disponibles pour sa mise en œuvre et la qualité de la coordination

\section{Figure 2}

\section{Liste des critères du formulaire de pointage de Preffi 2.0}

\section{Conditions contextuelles}

1 Conditions et faisabilité contextuelles

1.1 Soutien/engagement

1.2 Capacité

1.3 Leadership

1.3a Expertise et caractéristiques du chargé de projet 1.3b Point de mire pour le leadership

Analyse

2 Analyse des problèmes

$2 a$ Nature, gravité, ampleur du problème

2b Répartition du problème

2c Perception du problème par des intervenants

3 Déterminants du problème (psychologique), comportement et milieu

3a Modèle théorique

3b Contribution des déterminants au problème, comportement ou facteur environnemental

3c Susceptibilité des déterminants au changement

3d Priorités et sélection

\section{Choix et élaboration d'interventions}

4 Groupe cible

4a Caractéristiques générales et démographiques du groupe cible

4b Motivation et possibilités du groupe cible

4c Accessibilité du groupe cible

5 Cibles

5a Les objectifs correspondent à l'analyse

5b Les objectifs sont spécifiques, précisés dans le temps et mesurables

5c Les objectifs sont acceptables

$5 d$ Les objectifs sont réalisables

6 Élaboration de l'intervention

6.1 Raison d'être de la stratégie d'intervention

6.1a Adaptation des stratégies et des méthodes aux objectifs et aux groupes cibles

6.1b Expérience antérieure avec la ou les interventions

6.2 Durée, intensité et échéancier

$6.2 a$ Durée et intensité de l'intervention 6.2b Moment de l'intervention

6.3 Adaptation au groupe cible

6.3a Participation du groupe cible

6.3b Adaptation à la « culture »

6.4 Techniques efficaces (recommandées)

Place pour une approche personnalisée

Rétroaction sur les effets

Utilisation de stratégies de récompense

Suppression des obstacles au comportement de prédilection

Mobilisation de soutien/engagement social

Compétences en formation

Dispositions pour le suivi

Établissement d'objectifs et intention de la mise en cuvre Approche interactive

6.5 Faisabilité dans la pratique existante

6.5a Adaptation aux groupes cibles intermédiaires

6.5b Caractéristiques de la capacité de mise en œuvre de la ou des interventions

6.6 Cohérence des interventions/activités

6.7 Test préliminaire

Mise en cuvre

7 Mise en cuure

7.1 Choix d'une stratégie de mise en œuvre adaptée aux intermédiaires

7.1a Mode de mise en cuuve : de haut en bas ou de bas en haut

7.1b Adaptation des interventions de mise en œuvre aux intermédiaires

7.1c Acceptation des fournisseurs par les intermédiaires

7.2 Suivi et rétroaction

7.3 Intégration à la structure existante

Évaluation

8 Évaluation

8.1 Clarté et accord sur les principes d'évaluation

8.2 Évaluation du processus

8.3 Évaluation des effets

8.3a Un changement a-t-il été mesuré?

8.3b Le changement a-t-il été causé par l'intervention? 8.4 Rétroaction aux intervenants 
assurée par le chargé de projet. Les flèches qui pointent vers l'intérieur indiquent les moments auxquels des choix doivent être faits. La flèche pointant vers le choix d'une intervention est plus grosse pour indiquer que c'est à ce point que l'influence des conditions contextuelles se fait souvent particulièrement sentir.

Les 39 critères de qualité (indicateurs prévisionnels des effets) inclus dans Preffi 2.0 ont été sous-divisés en éléments (voir la figure 2). Les éléments du côté gauche portent principalement sur l'élaboration systématique d'une intervention, alors que ceux de droite portent particulièrement sur les aspects de la mise en œuvre. La structure fondamentale est la même que celle de Preffi 1.0, mais certains éléments ont été redéfinis l'un par rapport à l'autre. Au niveau de chacun des critères, le Preffi 2.0 a été révisé à fond tel que décrit précédemment et le nombre de critères a été ramené de 49 à 39.

\section{Cas 1 Exemple de l'opérationnalisation et des normes d'un critère Preffi}

6.3.a. Participation du groupe cible

Opérationnalisation:

1. Dans le cas d'interventions élaborées ailleurs (p. ex., au niveau national) : le groupe cible général a-t-il au moins été consulté pendant que l'intervention était élaborée ?

2. Pour n'importe quel projet: le groupe cible particulier pour le projet actuel (c.-à-d. les résidents du district cible) ont-ils au moins été consultés pendant que l'intervention était élaborée ou avant que le modèle d'intervention ait été choisi ?

3. Pour n'importe quel projet: compte tenu de la nature du projet, le groupe cible a-t-il participé suffisamment à l'élaboration ou à la sélection de l'intervention?

Normes :

- Faible : question $1=$ non ou sans objet et question 2 = non (ce qui rend la question 3 sans objet)

- Modérée : question 1 et/ou 2 = oui et question $3=$ non

- Forte : question 1 et/ou 2 = oui et question $3=$ oul

\section{Méthode de pointage}

Chaque critère de Preffi a été opérationnalisé au moyen d'une ou plusieurs questions particulières auxquelles il faut répondre par oui ou par non. Les réponses à ces questions permettent aux utilisateurs de noter comme " faible ", " modérée " ou " forte " la façon dont une intervention satisfait à un critère particulier (voir l'exemple de l'encadré 1).

Cette opérationnalisation a pour objet de fournir aux utilisateurs de Preffi un outil qui leur permet d'évaluer un programme de la façon la plus objective possible. Cependant, la nature d'un critère particulier ou des questions qui opérationnalisent ce critère peuvent ne pas toujours permettre une évaluation objective. À cet effet, on peut établir grosso modo une distinction selon trois types de critères. Le premier type comprend des critères pour lesquels les questions appellent une réponse sans équivoque, par exemple, "Sait-on dans quelle mesure le groupe cible perçoit effectivement le problème comme tel ? " (2.3). La deuxième catégorie sont des critères pour lesquels il n'existe pas de réponse directe, parce qu'ils exigent l'évaluation de certains aspects, tels que l'expertise du chargé de projet (1.3.a), ou si le groupe cible perçoit l'intervention comme étant compatible avec sa culture (6.3.b). Dans de tels cas, il est conseillé aux utilisateurs de rechercher le consensus des pairs au sujet de la question. Enfin, il y a un certain nombre de critères qui exigent essentiellement un avis d'expert. Citons pour exemple l'opérationnalisation du " modèle théorique " (3.1), qui comprend non seulement la question au sujet de l'utilisation ou non d'un modèle théorique, mais pour laquelle on demande également si l'on a établi comme plausible que le modèle choisi convient à une application dans une situation particulière.

Un certain nombre de critères de Preffi permettent de choisir l'option « incapable d'évaluer "; il s'agit en particulier des critères qu'il est difficile pour des tiers d'évaluer en se fondant sur la preuve documentaire fournie ; par exemple, l'expertise et les caractéristiques du chargé de projet (1.3.a). Dans d'autres critères, le manque d'information est évalué comme "faible ". Le formulaire de pointage permet de donner à chaque élément une note globale entre 1 et 10 , qui doit être composée à partir des notes attribuées individuellement à chaque critère au sein de l'élément. La note globale pour l'ensemble du projet est donc fondée sur les évaluations des éléments individuels. La note générale n'est pas nécessairement une simple moyenne, car des pondérations différentes peuvent être attribuées à certains critères d'un élément. Le verso du formulaire de pointage comporte un espace pour reporter les notes des éléments sous forme graphique et pour signaler les éléments devant être améliorés ainsi que les mesures qu'il faut prendre pour apporter ces améliorations.

\section{Expériences avec Preffi 2.0}

Une mise à l'essai, pouvant être considérée comme un pre-test de Preffi 2.0 a été réalisée auprès de 35 praticiens (étape 7), et a produit une évaluation favorable de la version provisoire (Meurs, 2002). 28 répondants sur 35 avaient travaillé avec Preffi 1.0 auparavant, dont 25 ont considéré Preffi 2.0 comme une amélioration, particulièrement en raison de l'amélioration de l'information qui le sous-tend et de l'opérationnalisation des critères de qualité. L'outil a obtenu une note générale moyenne de 7,7 sur 10 . Il a été évalué par la grande majorité comme étant valable, complet, clair, bien organisé et novateur. La plupart des répondants ont indiqué que Preffi 2.0 était utile pour eux-mêmes ( $83 \%$ ) et pour leurs collègues (89\%), tant sur le plan de l'élaboration de projets (79\%) que sur celui de leur évaluation (85\%). Ils considéraient Preffi 2.0 comme étant difficile et long, plutôt que facile et concis. Ils ont également signalé que le temps qu'ils devaient consacrer à l'appliquer diminuait à chaque application successive : le premier projet exigeait en moyenne 113 minutes pour l'évaluation, le deuxième 85 minutes. La pratique a de plus montré qu'un utilisateur aguerri de Preffi 2.0 peut évaluer entièrement un projet en une heure.

Il est devenu évident que les personnes utilisent Preffi à diverses étapes des projets, pour examiner leur propre projet et comme base pour étudier ceux de collègues. L'évaluation des projets d'autres personnes peut cependant être 
difficile si l'information manque ou est peu claire. Il a été établi qu'il faut chaque fois, en plus d'évaluer les documents fournis, avoir en plus une discussion avec le chargé de projet. Les utilisateurs trouvent que ceci produit un aperçu équilibré et utile des éléments du projet qui doivent être améliorés.

Les avis exprimés lors de l'étude pilote ont été corroborés par l'expérience avec notre programme de mise en œuvre de Preffi 2.0, dans le cadre duquel plus de 400 spécialistes de la promotion de la santé ont déjà été formés.

\section{Conclusions}

Pour être efficace, la promotion de la santé exige l'élaboration et la diffusion de programmes dont l'efficacité a été démontrée, ainsi que la connaissance des principes qui influent sur cette efficacité. Preffi est un système d'apprentissage dynamique qui permet de concourir à cet objectif de diverses façons. L'outil combine connaissances scientifiques et pratiques et sa fonction principale en est une de diagnostic de la qualité qui aide les utilisateurs à déterminer d'éventuelles améliorations à leurs projets. En sus, il pourrait éventuellement être utilisé comme outil de sélection de projets, bien qu'il faille davantage de recherche et de développement pour cette fonction ; une étude actuellement en cours a justement pour objet d'examiner combien d'évaluateurs il faudrait pour obtenir des conclusions dignes de confiance à cet égard.

Le perfectionnement de l'outil Preffi se poursuit, car de nouvelles versions sont produites régulièrement et son aspect pratique, sa fiabilité et sa validité sont sans cesse améliorés.

Les praticiens néerlandais sont d'avis que l'utilisation de Preffi pour évaluer leurs propres projets et ceux de leurs collègues produit actuellement une amélioration de la qualité des projets. Nous essayons à l'heure actuelle de corroborer cet avis au moyen de recherches plus systématiques.

\section{Références}

Bartholemew, L. K., Parcel, G. S., Kok, G. \& Gottlieb, N. H. (2001). Intervention mapping: designing theory- and evidence-based health promotion programs. Mountain View: Mayfield.

Boddy, D. (Ed.) (1999). The evidence of health promotion effectiveness; shaping public health in New Europe. Paris-Luxembourg: UIPES et Commission européenne.

Durlak, J. A. \& Welsh, A. M. (1997). Primary prevention mental health programs for children and adolescents: A meta-analytic review. American Journal of Community Psychology, 25, 115-152.

Glanz, K., Lewis, F. M. \& Rimer, B. K. (Eds.) (2002) Health behavior and health education: theory, research and practice. San Francisco: Jossey-Bass.

Jong, M. D. T. d. \& Schellens, P. J. (2000) Formatieve evaluatie. In Schellens, P. J., Klaassen, R. and Vries, S. d. (eds), Communicatief ontwerpen. Methoden, perspectieven en toepassingen. Assen: Van Gorcum.

Koelen, M. A., Vaandrager, L. \& Colomèr, C. (2001) Health promotion research: dilemmas and challenges Journal of Epidemiology and Community Health, 55 , 257-262.

Kok, G. J., Borne, B. v. d. \& Mullen, P. D. (1997) Effectiveness of health education and health promotion: meta-analyses of effect studies and determinants of effectiveness. Patient Education and Counseling, 30, 19-27.

McQueen, D. V. \& Anderson, L. M. (2002) What counts as evidence: issues and debates on evidence relevant to the evaluation of community health programs. In Rootman, I., Goodstadt, M., McQueen, D., Potvin, L., Springett, J. and Ziglio, E. (eds), Evaluation in Health Promotion: principles and perspectives. Copenhague: OMS/EURO.

Meurs, L. H. v. (2002) Concept Preffi 2.0; reliability and usefulness, document de recherche (en Néerlandais). Woerden: NIGZ.

Molleman, G. R. M. (1999) Implementing the Preffi: the use of guidelines for practitioners in the Netherlands in Best Practices, a selection of papers on Quality and Effectiveness in Health Promotion. Helsinki/Talinn: Centre finlandais de promotion de la santé.

Molleman, G. R. M., Hosman, C. M. H., Kok, G. J. \& Peters, L. W. H. (2004) Implementation of a quality assurance instrument (Preffi 1.0) to improve the effectiveness of health promotion in the Netherlands submitted.

Molleman, G. R. M., Peters, L. H. M., Hommels, L. H. \& Ploeg, M. A. (2003) Assessment Package; Health Promotion Effect Management Instrument Preffi 2.0 .Woerden: NIGZ.

Disponible à l'adresse: http://www.nigz.nl/english/index.cfm?code=904

Peters, L. H. M., Molleman, G. R. M., Hommels, L. H., Ploeg, M. A., Hosman, C. M. H. \& Llopis, E. (2003) Explanatory Guide Preffi 2.0. Woerden: NIGZ.

Disponible à l'adresse: http://www.nigz.nl/english/index.cfm?code=904 